\title{
Situaciones de docencia: una alternativa para evaluar competencias en posgrados
}

\author{
Ana María González Ortiz
}

Profesora investigadora

Centro de Investigación y Docencia

\section{Resumen}

$\mathrm{E}$ 1 artículo que se presenta contiene el estudio realizado en torno a la identificación de competencias docentes así como la construcción y validación de un instrumento dirigido a los estudiantes para evaluar las competencias siguientes: interacción pedagógica, comunicación educativa y evaluación de los aprendizajes, en tres instituciones de posgrado. Para la construcción del cuestionario se tomaron competencias que por sus niveles de desempeño, se pudieran valorar desde la visión de los estudiantes como participantes de los procesos de enseñanza y aprendizaje.

El trabajo comprendió dos etapas: en la primera se realizó la definición del perfil docente y la caracterización de la docencia a través de un diagnóstico, utilizando un cuestionario dirigido a 33 docentes.

La segunda consistió en la identificación de competencias docentes y la construcción y piloteo del cuestionario. El procedimiento metodológico se realizó con un panel de expertos a través del método DACUM (Disegn a curriculum) y las técnicas TKJ y Delphi que permitieron la generación de ocho competencias relacionadas con la práctica docente y educativa. Cada una de las competencias comprende: nombre, descripción, componentes, indicadores y evidencias de desempeño. La construcción del cuestionario comprende 28 situaciones de docencia representadas en tres niveles de desempeño: experto, con cierta experiencia y novato a través de rúbricas que permiten una evaluación del desempeño centrada en aspectos cualitativos, con niveles de progresión de un proceso que supera la medición de reactivos. Ambas etapas permitieron ubicar en el contexto, la práctica docente de los profesores de los posgrados, las competencias que ponen en práctica al desempeñar su función, así como aquellas que se identifican y evalúan, lo cual constituye la oportunidad de desarrollarlas a través de procesos de formación docente. 
REVISTA DE INVESTIGACIÓN EDUCATIVA DE LA REDIECH N. 3

Palabras clave: Competencias, evaluación, docencia, competencias docentes.

\section{Introducción}

La docencia representa una actividad compleja y multideterminada dado que en ella convergen varios factores, uno de ellos es la política educativa desde la cual se emiten directrices que intervienen en su desarrollo. En la actualidad la tendencia del enfoque basado en competencias forma parte de los currículos de los diferentes niveles educativos, en los que se señala el sustento pedagógico y didáctico del aprendizaje, la enseñanza y la evaluación.

En la formación profesional se ha incorporado dicho enfoque de manera que quienes promueven el desarrollo de competencias en los estudiantes, a su vez desarrollen las propias. $\mathrm{Si}$ bien las competencias que los profesores requieren para su desempeño se han señalado desde organismos internacionales y se han asumido en México a partir de las reformas educativas de los últimos 20 años, la identificación de las mismas y la manera de evaluarlas situadas en la práctica docente, constituyen un aspecto importante a considerar en las estrategias de desarrollo profesional.

En la revisión de la literatura se rescatan aportaciones significativas para la realización de este estudio. Respecto a la definición del perfil docente e identificación de competencias en educación superior, se evidencia la participación de diversos actores para analizar la concepción de competencia y su relación con el aprendizaje, quienes presentan registros sobre cómo elaboran grupalmente su realidad y relatan experiencias, asumiendo que las competencias son el resultado de la integración dinámica de distintos tipos de conocimientos y prácticas (saberes).

Por su parte, la Red de Investigadores sobre Evaluación de la Docencia (RIED) desarrolló el Modelo de Evaluación de Competencias Docentes (MeCD) para educación media superior y el Modelo de evaluación/formación. El primero comprende una dimensión para evaluar el contexto institucional (filosofía, misión, visión, trabajo en claustros) y tres para evaluar la práctica docente: planeación del proceso enseñanza-aprendizaje; conducción de secuencias didácticas y valoración del impacto del proceso enseñanza-aprendizaje. Por su parte el segundo, identifica tres grandes momentos que por su dinámica son secuenciados y a la vez paralelos: las actividades que se desarrollan previas al proceso de enseñanza-aprendizaje, las que se cubren mientras se conduce dicho proceso y aquellas que se llevan a cabo como una valoración del impacto del proceso de enseñanza y aprendizaje.

Se considera que estas tres dimensiones coexisten en un contexto institucional determinado, donde los factores filosóficos, epistemológicos, psicológicos, sociales y pedagógicos tienen su razón de ser. Finalmente es necesario mencionar que uno de los ejes rectores que orientan al modelo son las competencias docentes (García, Cabrero, Loredo, Luna y Rueda, 2008). Por ello, toman en cuenta diversos niveles de competencia en el desempeño reconociendo que existen diferencias en concepciones, creencias y prácticas de enseñanza.

En la educación superior, las Instituciones Formadoras de Docentes (IFD) que ofertan posgrados en el estado de Chihuahua, tienen una particularidad distintiva frente a las uni- 
versidades: sus estudiantes son profesores adscritos generalmente a la educación básica. Este rasgo, hace necesaria la implementación de estrategias de enseñanza y aprendizaje que vayan más allá de técnicas de estudio, transmisión de conocimientos y uso de los medios tecnológicos, se requiere de un docente que tenga plena conciencia de los alcances su intervención educativa, y del compromiso que tiene como educador.

\section{Referentes teóricos}

Identificar la docencia como una tarea compleja, implica reconocer que su evaluación representa tomar posición en alguno de los diversos enfoques de enseñanza, actualmente desde competencias se "ofrece la oportunidad de replantear las funciones delegadas al profesor y por lo tanto, dan cabida a nuevos desafíos para su formación permanente y desarrollo profesional que, sin duda impactarán claramente la manera de hacer la futura evaluación del desempeño" (Rueda, 2008, p. 32).

La docencia como práctica social trasciende lo educativo, en la mediación que se establece entre los sujetos participantes está presente el conocimiento como producción social (Gorodokin, 2008).

Para abordar el actual enfoque del plan y programas de estudio, es importante el análisis respecto a la noción de competencia, dado que no proviene de un solo paradigma teórico, ni de una sola tradición psicopedagógica, algunos autores identifican que las definiciones que coinciden con la idea de la movilización de recursos como el eje central del concepto de competencia, tienen implícito el elemento contextual, referido al momento de aplicar estos saberes e implican conocimientos interrelacionados con habilidades y actitudes (Zabala y Arnau, 2008).

De acuerdo con Marín (2003), una persona logra desarrollar una competencia cuando ante un contexto, "situación" o problema concreto, se "desempeña" de manera adecuada (nivel de logro) movilizando de forma integral conocimientos, procedimientos y actitudes (recursos cognitivos) para encontrar nuevas soluciones.

Se coincide con Ibáñez (2007: 152) cuando señala que:

Dichas situaciones se distinguen de otras por constituir problemas por resolver. Una situación o tarea problema se refiere entonces a un determinado arreglo de eventos ambientales que demandan una respuesta del individuo que lo modifique hacia una dirección convencionalmente determinada y que normalmente constituye la solución del problema. La respuesta del individuo ante la situación problema -es decir, su desempeño- puede ser exitoso o no serlo; pero sólo cuando su desempeño es exitoso en forma consistente puede hablarse de competencia.

La evaluación de competencias representa un proceso de constatación de evidencias de desempeño y del conocimiento y comprensión que una persona demuestra en relación con una función definida, usualmente en un perfil o en una norma de competencia (Vargas, 2002). Ante esto, desde el enfoque de las competencias, la observación del desempeño es relevante para determinar el nivel de competencia (Hernández et al, 2005), entendiendo la evaluación como un juicio de valor que se emite al comparar la expresión real de la competencia frente a un grupo de criterios de desempeño o pará- 
metros establecidos idealmente en el perfil de desempeño.

Es importante reconocer que la evaluación puede favorecer la reflexión sobre la práctica docente y derivar procesos de formación continua que permitan un mejor desempeño.

\section{Procedimiento metodológico}

La reflexión que se produjo entre el análisis de la práctica docente y la caracterización de la misma, al tomar como referente una docencia por competencias, los modelos de evaluación de competencias docentes de la RiED, así como otros referentes teórico-conceptuales, constituyen los fundamentos de la propuesta para las IFD.

Metodológicamente se procedió con el método Design a Curriculum (DACUM) planteado por Mertens (1997), para la identificación de las competencias docentes. Su aplicación se realizó con ocho docentes expertos: académicos con experiencia (directivos y fundadores de los tres posgrados), conocimientos sobre la docencia $y$ el funcionamiento de instituciones de nivel superior. En el trabajo, se expusieron para su análisis y discusión, grandes áreas de desempeño docente inherentes al proceso educativo. El empleo de la técnica TKJ modificada, permitió recabar la opinión y el sentir de los participantes y consensuar las diversas perspectivas planteadas en torno a las competencias docentes de los posgrados. Se le solicitó a cada experto la aportación de cinco tareas docentes, que derivó en la integración de ocho competencias identificadas de manera cercana a las definiciones de los modelos de la Ried: diseño de la docencia, gestión de la progresión de los aprendizajes, interacción pedagógica, comunicación educativa, evaluación de aprendizajes, condiciones contextuales, formación continua y cultura organizacional, pero planteadas específicamente en función de la práctica docente de los profesores y las características académicas y de organización de las IFD.

Estas contemplan el aspecto contextual de las IES, así como los tres momentos de la práctica docente: lo previo, el trabajo docente en el aula y lo posterior a este proceso. Es así como, para la construcción del instrumento dirigido a alumnos, se tomaron en cuenta tres de las competencias relacionadas con la docencia y sus respectivos componentes: interacción pedagógica (objeto de conocimiento, ambientes de aprendizaje y relación educativa), comunicación educativa (estrategias de comunicación, herramientas de comunicación y recursos didácticos) y evaluación de los aprendizajes (aspectos de la evaluación, socialización y formas de evaluación).

Respecto de las otras cinco competencias se consideró que de acuerdo con las situaciones de docencia y las evidencias de desempeño que las integran, su evaluación tendría mayor significado al realizarse como evaluación de pares, directivos o autoevaluación, es decir complementarían una evaluación integral del desempeño docente, tarea pendiente de realizarse tomando como sustento el trabajo realizado en este estudio.

La valoración emitida por los expertos en cuanto a las competencias, sus situaciones de docencia y los desempeños docentes, se realizó con el análisis de las categorías a través de la técnica Delphi. En la primera revisión se aprobaron por consenso, la mayoría de las situaciones de docencia de las tres competencias. En la última 
ronda se efectuaron las modificaciones propuestas para los niveles que reflejaban las rúbricas.

La construcción del cuestionario se llevó a cabo de la siguiente forma:

- se realizó un mapeo para relacionar los indicadores y evidencias de cada competencia.

- se seleccionaron y redactaron las 28 situaciones de docencia para las tres competencias docentes.

- con esa base se diseñaron los desempeños docentes que permitieron la evaluación.

- se fijaron los criterios para evaluar el nivel de competencia.

- se construyeron las matrices de valoración o rúbrica para evaluar las situaciones de docencia de cada competencia.

El cuestionario dirigido a alumnos comprende cuatro bloques: en el primero y segundo se solicitan datos de la institución y del estudiante; el tercero, contiene las indicaciones y el cuarto presenta las situaciones de docencia que los estudiantes pueden identificar, para enseguida valorar, en tres niveles de desempeño docente, los comportamientos de los profesores ante una situación determinada: 14 conciernen a la competencia de interacción pedagógica; siete para comunicación educativa y siete evaluación de los aprendizajes.

El instrumento se sometió a un proceso de validación de contenido (mediante juicio de expertos). El modelo de fiabilidad empleado fue el de Alfa de Cronbach para determinar la consistencia interna a través de la correlación inter-elementos promedio. El instrumento aplicado a la muestra piloto permitió obtener su confiabilidad y poder de discriminación. En los análisis de fiabilidad del instrumento se observó que todos los valores son superiores a .846 para Alfa de Cronbach y .847 en Alfa de Cronbach basada en los elementos tipificados. De esta manera la confiabilidad del instrumento completo es mejor que la de las escalas breves.

Para evaluar la capacidad de discriminación del instrumento entre subgrupos (sexo, edad, instituciones y formación profesional), se emplearon Anova y pruebas " $t$ " de student, a fin de establecer diferencias estadísticas entre subgrupos con nivel de significancia adecuados.

También se utilizó el análisis de correspondencia múltiple el cual permite determinar la posición (distancia) de los objetos/sujetos (segmentos, grupos, sectores y clasificaciones) en una serie de atributos, características, escalas de valoración y cualidades, entre otras, en un espacio vectorial de dos, tres o más dimensiones.

El instrumento fue piloteado, aplicándose a 447 estudiantes (cada alumno evaluó a tres o cuatro profesores simultáneamente), valorando en total las competencias de 37 docentes de los tres posgrados. Estos, representan el $60 \%$ de la planta docente adscrita a estas instituciones.

La construcción del cuestionario dirigido a alumnos para evaluar las competencias docentes en los posgrados de las IFD, consideró los elementos de la definición de competencia: capacidad para movilizar recursos cognitivos en una situación específica, contienen situaciones de docencia de cada competencia docente evaluada, a su vez, las rúbricas permiten una valoración del desempeño centrada en aspectos cualitativos que reflejan niveles progresivos de acercamiento al enfoque por competencias y hacen posible identificar niveles de desempeño. 
REVISTA DE INVESTIGACIÓN EDUCATIVA DE LA REDIECH N. 3

Esta propuesta de evaluación de competencias busca trascender el uso de cuestionarios diseñados a través de escalas Lickert con reactivos que señalan la frecuencia de un determinado comportamiento, situación recurrente que se identificó en la revisión de estudios realizados para evaluar la docencia.

\section{Resultados del piloteo}

A partir de las condiciones profesionales y laborales en las cuales los profesores de los posgrados se incorporaron a las IFD, el personal docente que participó en la fase de diagnóstico, presenta características de su identidad personal y profesional (Cuadro 1) que permean la práctica docente.

\section{Cuadro 1. Perfil docente}

\begin{tabular}{|c|c|c|c|c|c|c|c|c|}
\hline Docentes & $\begin{array}{c}\text { Promedio } \\
\text { de Edad }\end{array}$ & $\mathrm{H}$ & $\mathrm{M}$ & $\begin{array}{c}\text { SEP } \\
\text { (promedio) }\end{array}$ & $\begin{array}{c}\text { Nivel } \\
\text { superior } \\
\text { (promedio) }\end{array}$ & $\begin{array}{c}\text { Docencia } \\
\text { en el nivel } \\
\text { superior } \\
\text { (Promedio) }\end{array}$ & $\begin{array}{c}\text { Nivel educativo } \\
\text { de procedencia }\end{array}$ & $\begin{array}{c}\text { Institución } \\
\text { donde cursó el } \\
\text { posgrado }\end{array}$ \\
\hline 33 & $\begin{array}{c}42 \\
\text { años }\end{array}$ & 17 & 16 & $\begin{array}{c}22 \\
\text { años }\end{array}$ & 9 años & 10 años & $\begin{array}{c}29 \text { Educación } \\
\text { básica } \\
2 \text { media } \\
\text { superior } \\
2 \text { superior }\end{array}$ & 24 IFD \\
\hline
\end{tabular}

En la identidad profesional que se construye, intervienen concepciones y estilos de trabajo docente que definen a las propias instituciones y a los sujetos. Como señala Medina (2000), en el ejercicio de la docencia se recrean las historias personales, el origen social, expectativas de formación y de escolaridad, el ejercicio profesional y el tipo de relaciones que median la práctica del profesor.

Referido al nivel de desempeño docente al compararlo por sexo, los resultados encontrados sugieren que no hay diferencias (gráfica 1), con excepción de las siguientes situaciones de docencia:

Los docentes hombres (63.2\%) así como las mujeres (36.8\%), en la evaluación de las situaciones de docencia que corresponden a las competencias de "interacción pedagógica" y "evaluación de los aprendizajes", se ubican en el nivel con cierta experiencia. El hecho de que no se presenten diferencias en estas competencias, significa que los estudiantes no establecen distinciones en el desempeño docente tomando como referencia la pertenencia a uno y otro sexo, lo cual implica que en este nivel educativo la función docente no está marcada por estereotipos de género en los que se señalan distinciones de acceso a la "ciencia". 


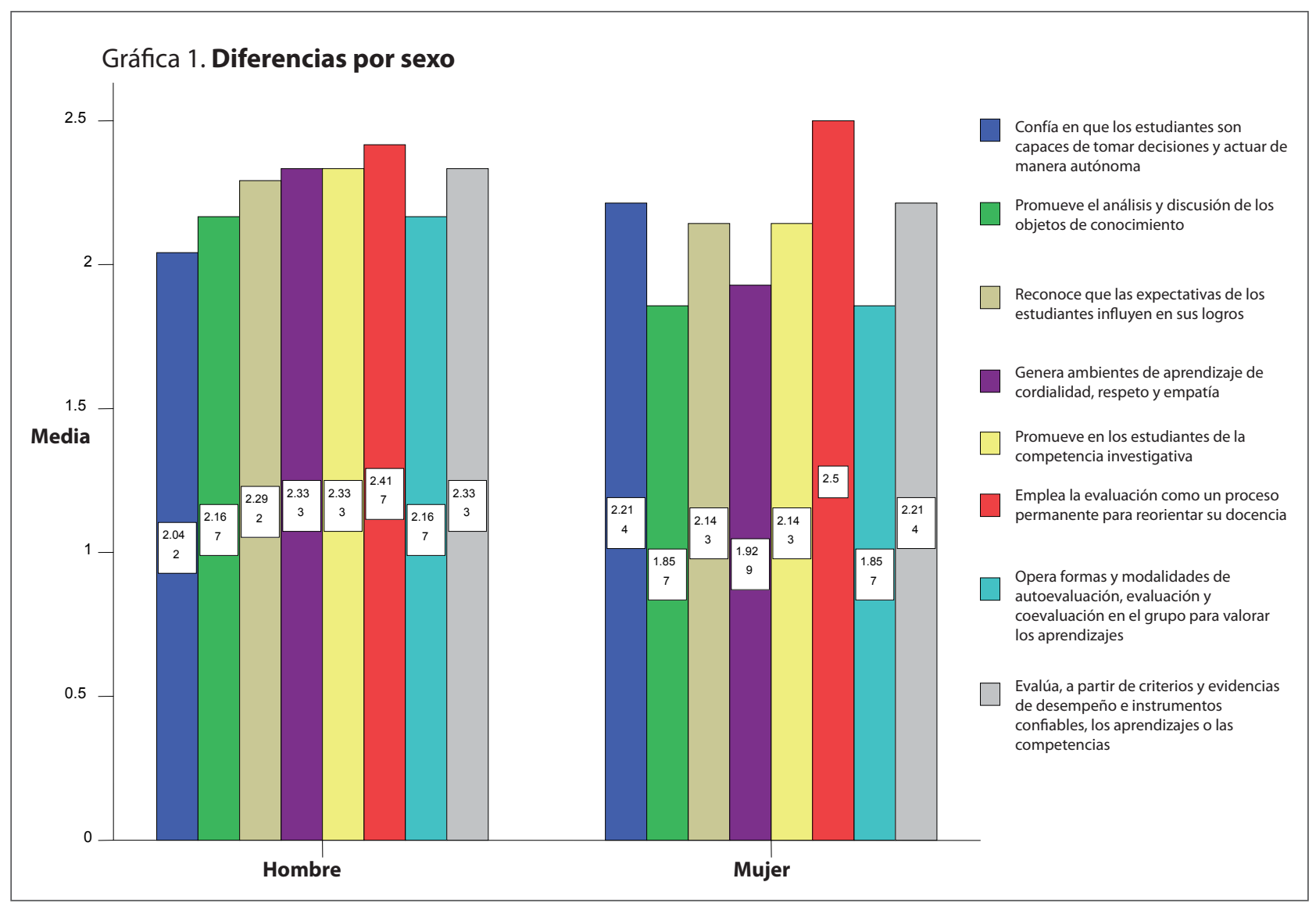

\section{Antigüedad docente}

De igual manera, no presentan diferencias por rangos de antigüedad docente, salvo las siguientes situaciones que corresponden a la competencia de interacción pedagógica (Gráfica 2).

En el desarrollo de la función docente, se adquieren conocimientos y experiencias, de acuerdo a los resultados es importante resaltar que los docentes de más de 10 años, favorecen la discusión de ideas tomando en cuenta la diversidad y pluralidad, lo que denota mayor apertura para tomar en cuenta a los demás, dejando de ser el centro de la interacción pedagógica.

\section{Edad de los docentes}

Los docentes participantes fueron agrupados de acuerdo a la edad por rangos: el 21.1\% tienen entre 31 a 40 años; el 39.5\% los de 41 a 50 años y el mismo porcentaje para los de 51 años o más. Las edades que representan los mayores porcentajes, pueden explicarse en el hecho de la ruta marcada para la profesionalización docente posterior a la normal básica, es decir los estudios de licenciatura y posgrado representan la inversión de tiempo y recursos que los profesores deben considerar para desempeñar la función docente en educación superior. 
REVISTA DE INVESTIGACIÓN EDUCATIVA DE LA REDIECH N. 3

\section{Gráfica 2. Diferencias por rango de antigüedad}

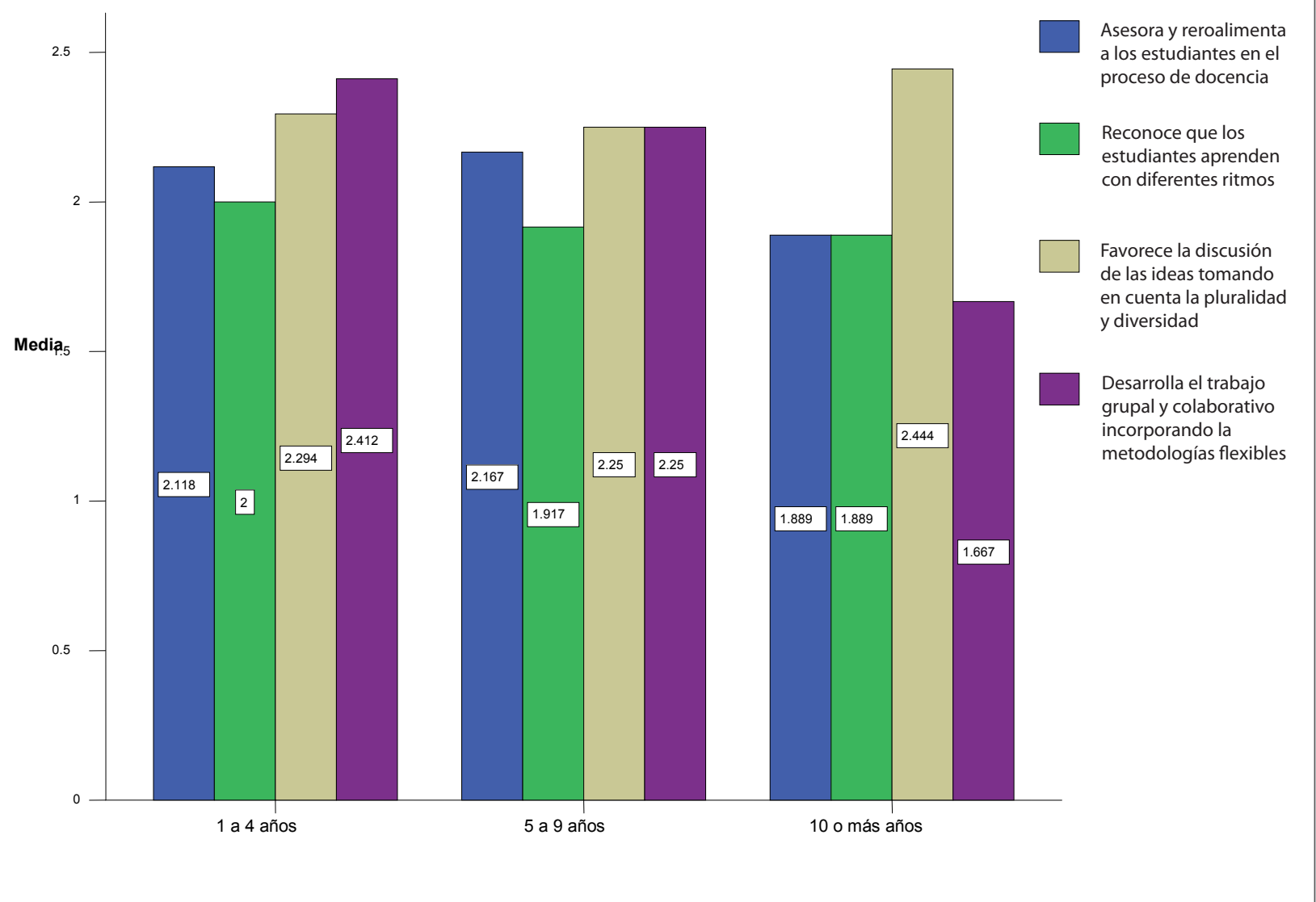

De acuerdo a los grupos de edad de los docentes evaluados, destacan las puntuaciones que obtienen en la competencia referida a la evaluación de los aprendizajes (gráfica 3), particularmente en la situación de docencia: acordar con los estudiantes, los criterios y formas de evaluación, en quienes tienen 51 años o más se ubican en el nivel de novato con 1.60, esto permite identificar que predomina la heteroevaluación.
De acuerdo a Zabalza (2007), una de las actividades docentes con más fuertes repercusiones en los estudiantes es la evaluación. Ésta se convierte en un factor primordial que incide en los aprendizajes y en la enseñanza pues si bien los objetivos, contenidos, manejo de recursos y metodologías, constituyen componentes importantes de la labor docente, las dimensiones formativa y de acreditación de la evaluación son el sustento de los currículos. 


\section{Gráfica 3. Diferencias por edad}

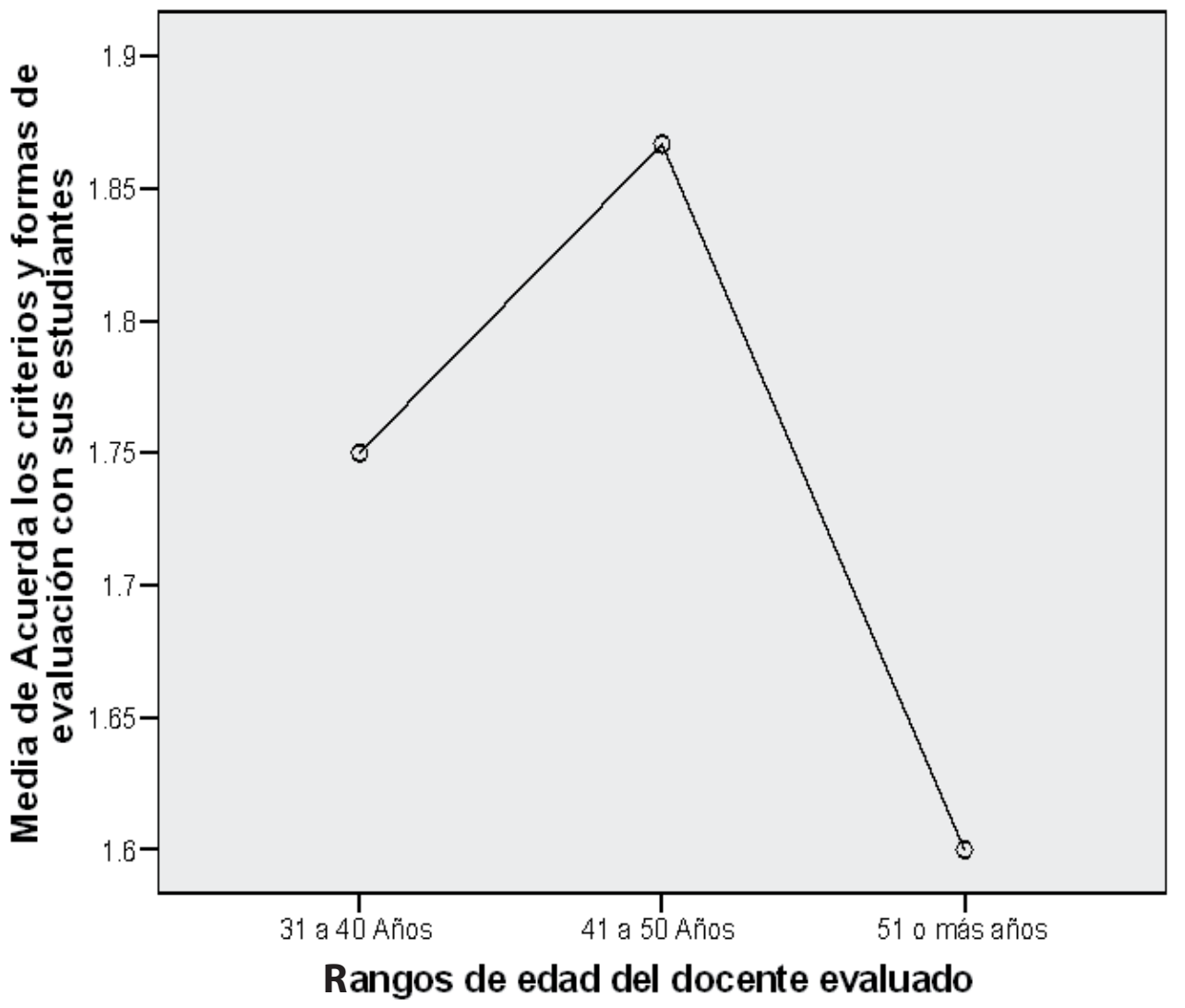

En general en esta competencia (gráfica 4), los docentes se ubican en el nivel con cierta experiencia lo cual hace evidente que esta competencia profesional es deficitaria, "sabemos poco, en general de la evaluación (...) y eso repercute fuertemente en la práctica docente" (Zabalza, 2007: 146).

En las situaciones de docencia: reconoce que las expectativas de los estudiantes influyen en sus logros, favorece la discusión de las ideas tomando en cuenta la pluralidad y diversidad y emplea correctamente el lenguaje oral en su discurso didáctico, son evaluados como expertos (2.63), quienes tienen entre 31 y 40 años.

Referente a las puntuaciones obtenidas en la competencia comunicación educativa, puede identificarse un proceso de cambio en la redefinición del papel centrado en el profesor hacia una autonomía en la que el alumno pueda integrar la información en estructuras con- 
Gráfica 4. Competencia: evaluación de los aprendizajes

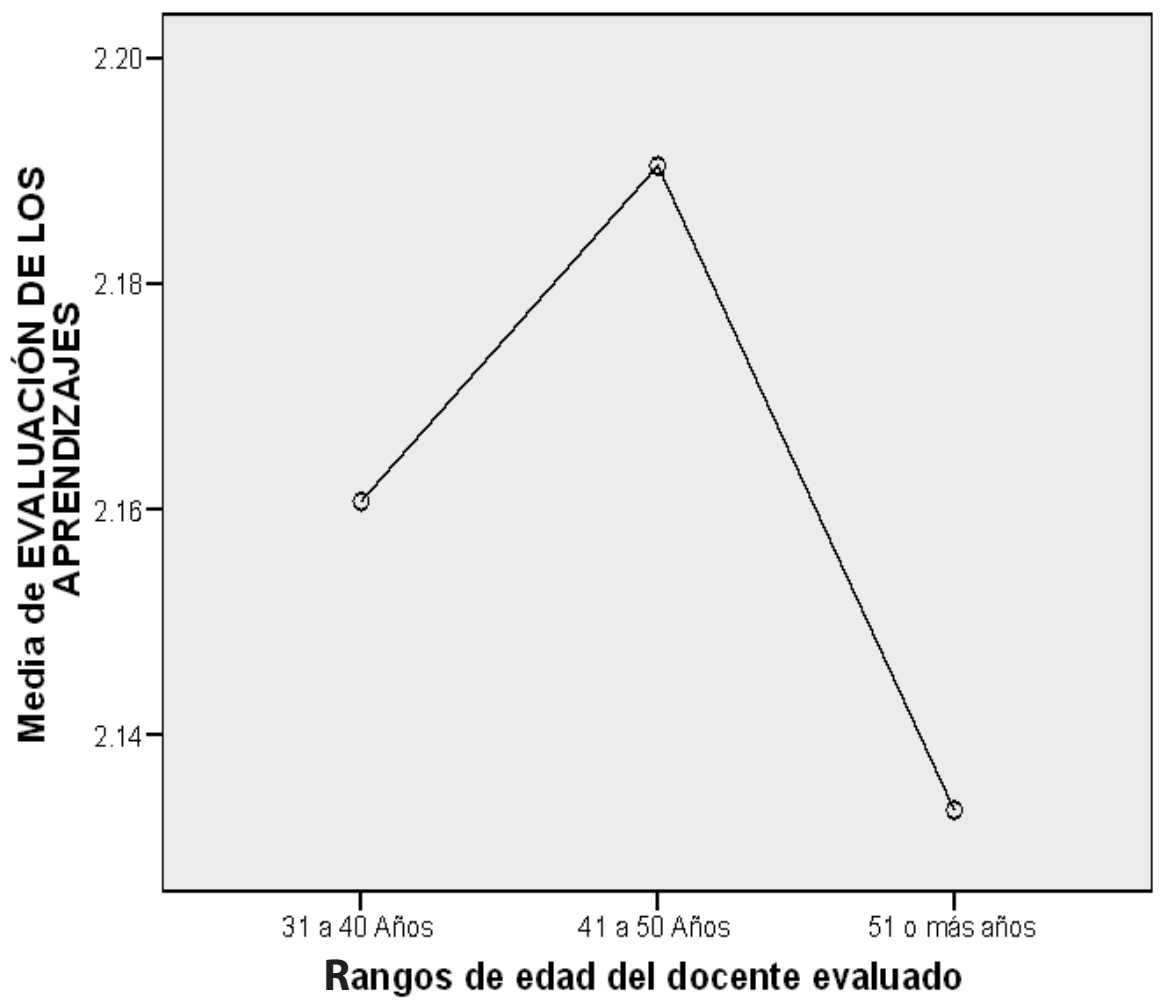

ceptuales útiles en su proceso de aprendizaje, como puede apreciarse en la gráfica 5, un alto porcentaje de la evaluación en esta competencia, permite ubicar a los docentes en el nivel de expertos.

De manera específica, la aportación de las tecnologías de la información y la comunicación en los procesos de enseñanza y aprendizaje, "podrían liberar al profesor de su papel de trasmisor" y permitirle el diseño de estrategias didácticas que impliquen un "auténtico reto cognitivo y que favorezcan la integración de los conocimientos adquiridos" (Zabalza, 2007: 94).
En la competencia interacción pedagógica en su componente relación educativa, son evaluados como expertos con 2.41, los docentes con 31 a 40 años de edad. En el resto de las competencias, se ubican en el nivel con cierta experiencia (gráfica 6).

\section{Antigüedad en el nivel superior}

La experiencia adquirida es también un aspecto importante del desempeño, pues se vivencia una multiplicidad de prácticas educativas que conjuntamente con la superación profesional, amplían el significado de la labor 
Gráfica 5. Competencia: comunicación educativa

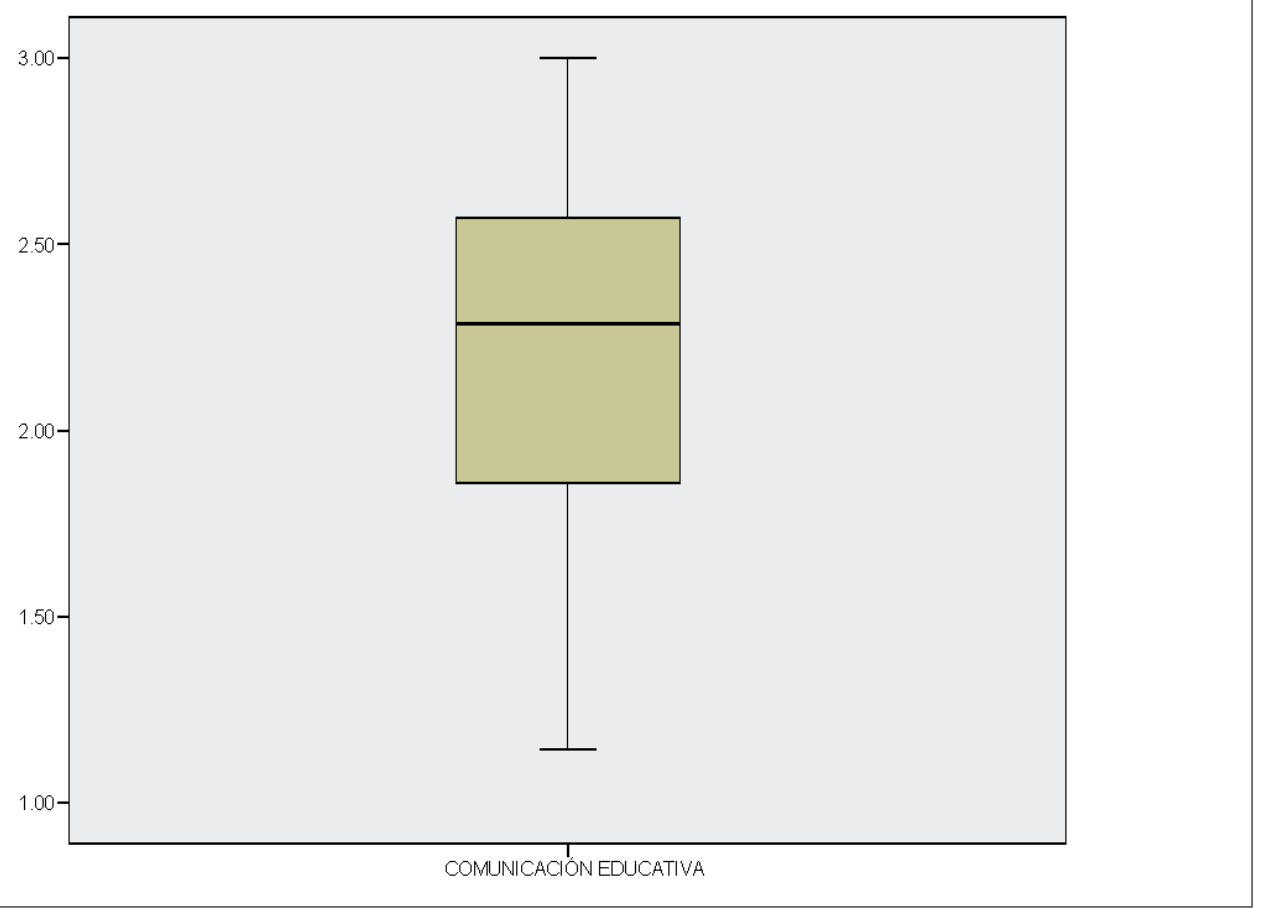

Gráfica 6. Competencias de interacción pedagógica, comunicación educativa y evaluación de los aprendizajes

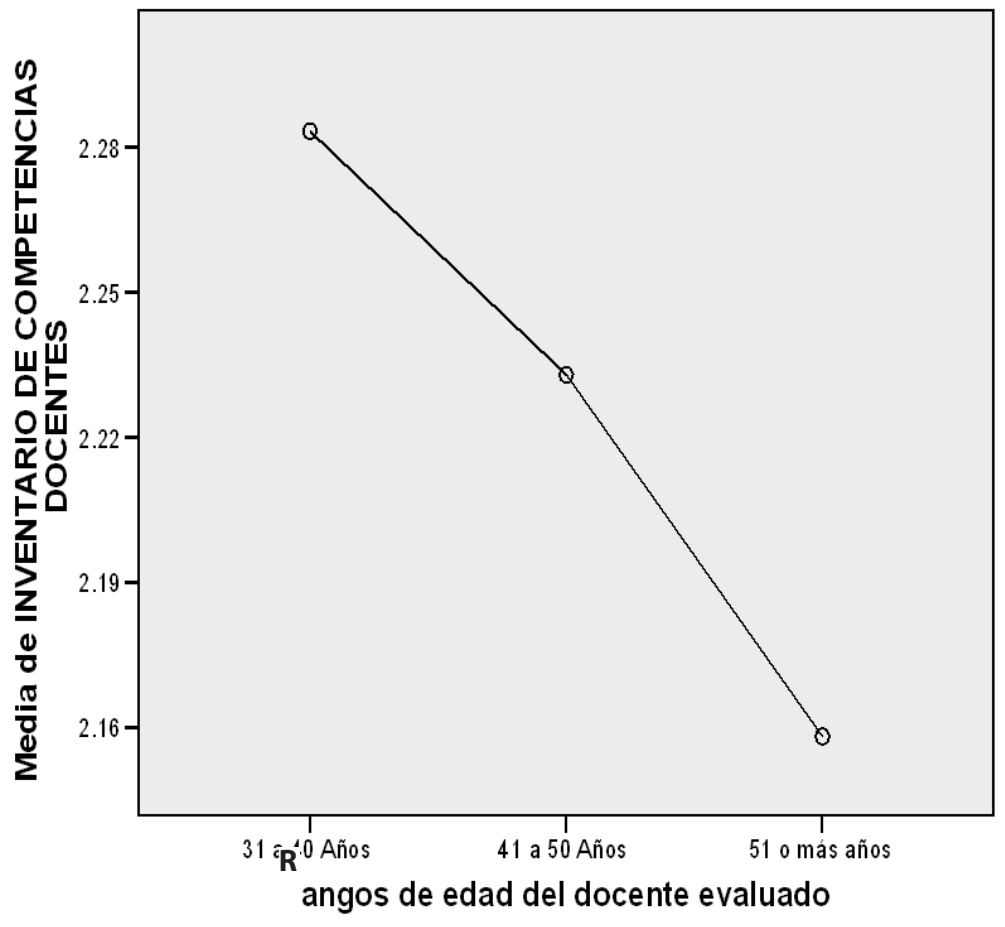


docente. Los docentes que tienen entre uno y cuatro años en las IFD, son evaluados en el nivel con cierta experiencia en las situaciones de docencia: promueve el trabajo centrado en el aprendizaje y en la relación educativa acepta críticas y sugerencias de los docentes

Estas diferencias se aprecian de manera más objetiva en el siguiente análisis de correspondencia múltiple (gráfica 7), que relaciona el inventario de competencias docentes con tres variables: sexo, nivel de estudio de los docentes evaluados y las instituciones a que pertenecen.

En lo referente a la competencia interacción pedagógica, la institución A se aproxima al nivel alto, la institución B es evaluada cercana al nivel 1 y la $\mathrm{C}$ en el 3. En los estudios realizados, son mejor evaluados quienes tienen doctorado, maestría y candidatos al grado de maestría. No se presentan diferencias significativas en la evaluación que reciben las y los docentes.

\section{Gráfica 7. Evaluación de la interacción pedagógica por sexo, institución y nivel de estudios}

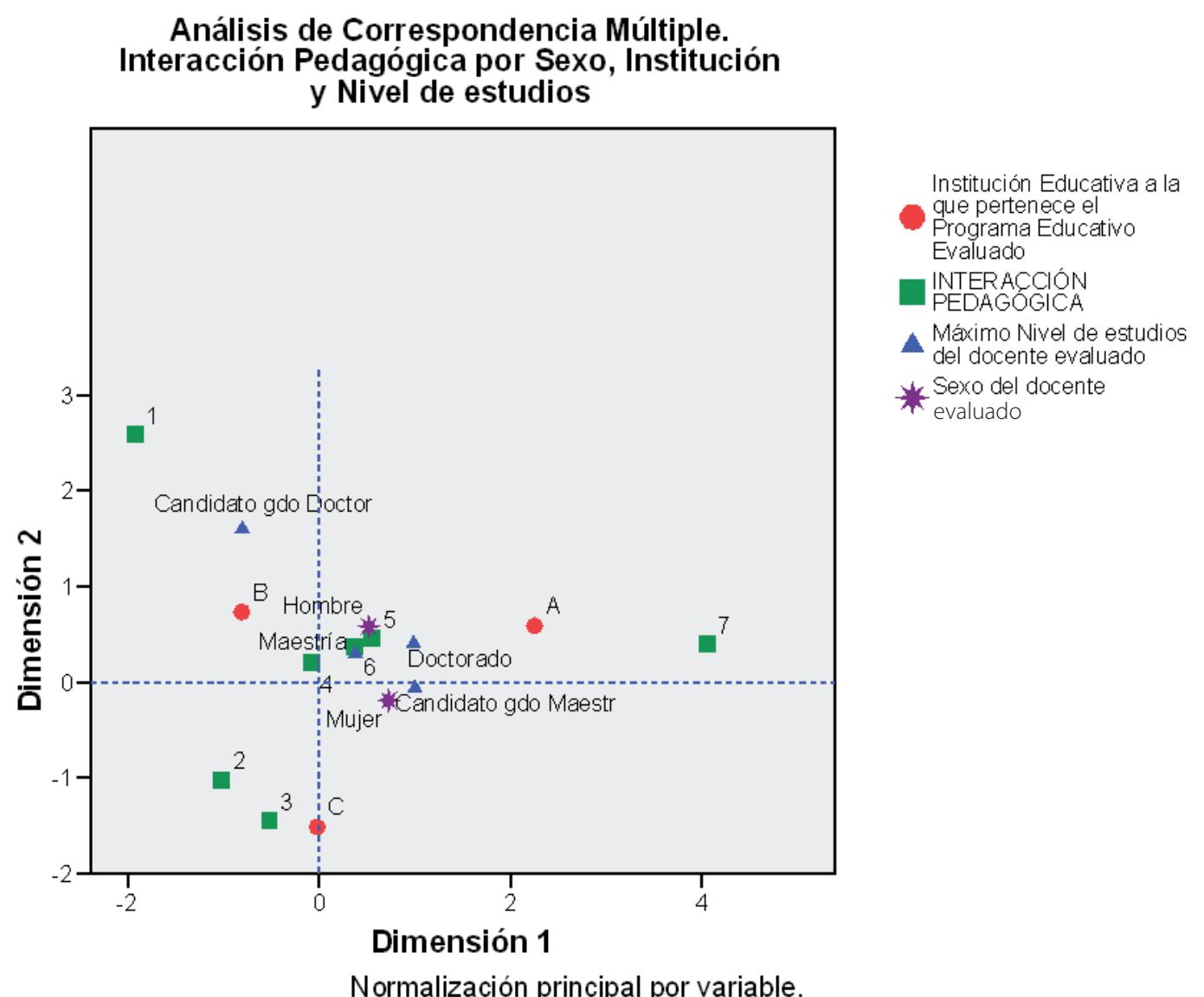

Normalización principal por variable. 


\section{Gráfica 8. Comunicación educativa por sexo, institución y nivel de estudios}

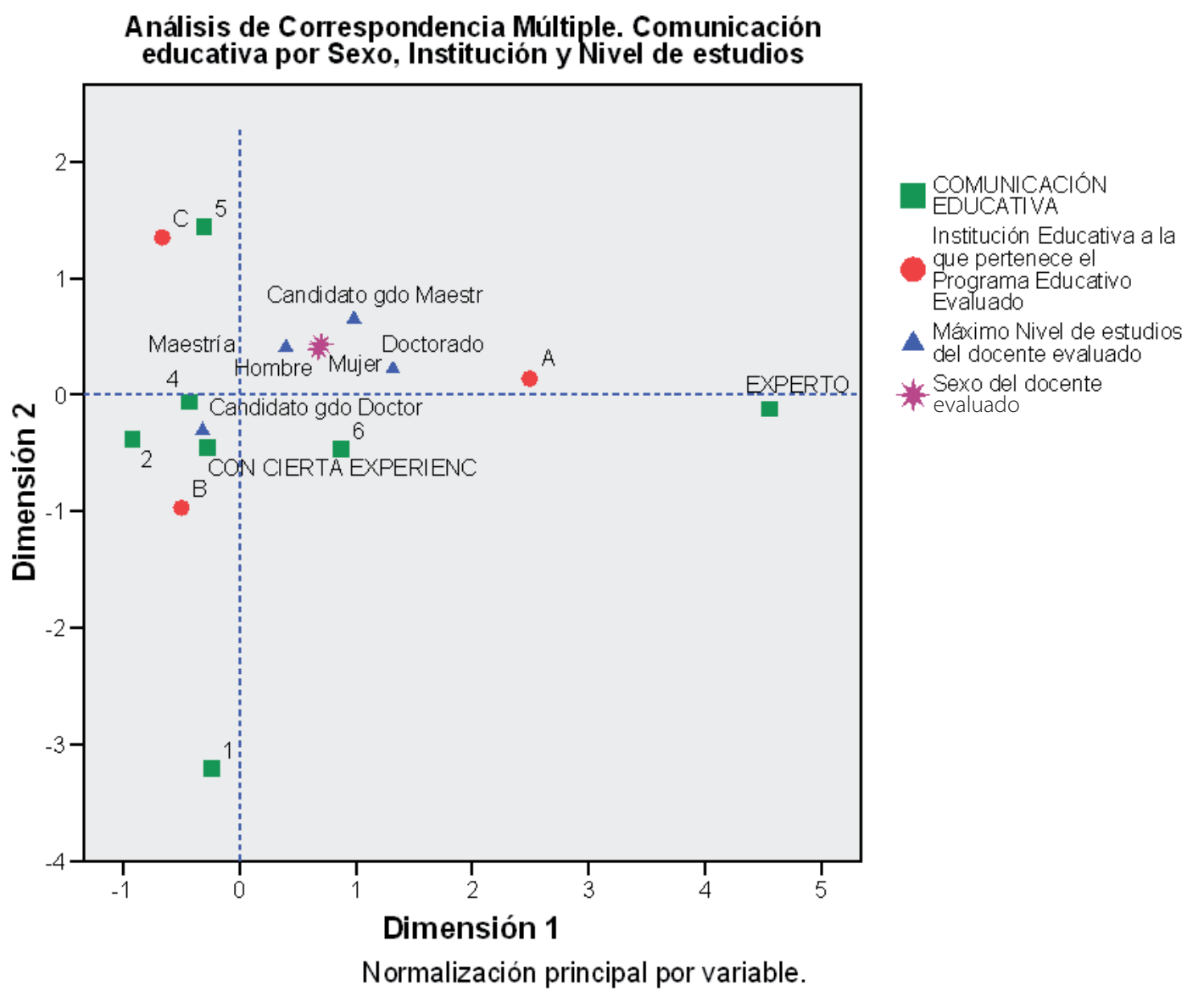

En la competencia comunicación educativa (gráfica 8), los docentes candidatos al grado de maestría, maestría y doctorado, se ubican en el nivel con cierta experiencia. En este mismo nivel están los docentes hombres y mujeres. Por otra parte la institución B tiene el nivel más bajo.

En esta competencia, la institución A es evaluada de manera más próxima al nivel alto (7) y la C se ubica en el bajo (1). Con respecto al nivel de estudios, quienes son candidatos al grado de doctor son evaluados en el nivel bajo
(2). En los niveles medios se ubican los y las docentes de acuerdo al sexo al cual pertenecen.

Como puede apreciarse, respecto de las instituciones evaluadas, en el mayor nivel de proximidad al inventario de competencias docente es la A. En contraposición, la institución C se ubica en los niveles 2 y 3 .

Por su parte, los docentes con doctorado, candidatos al grado de maestría y maestría son los mejores evaluados con respecto a quienes son candidatos a grado de doctor. 


\section{Gráfica 9. Inventario de competencias por sexo, institución y nivel de estudios}

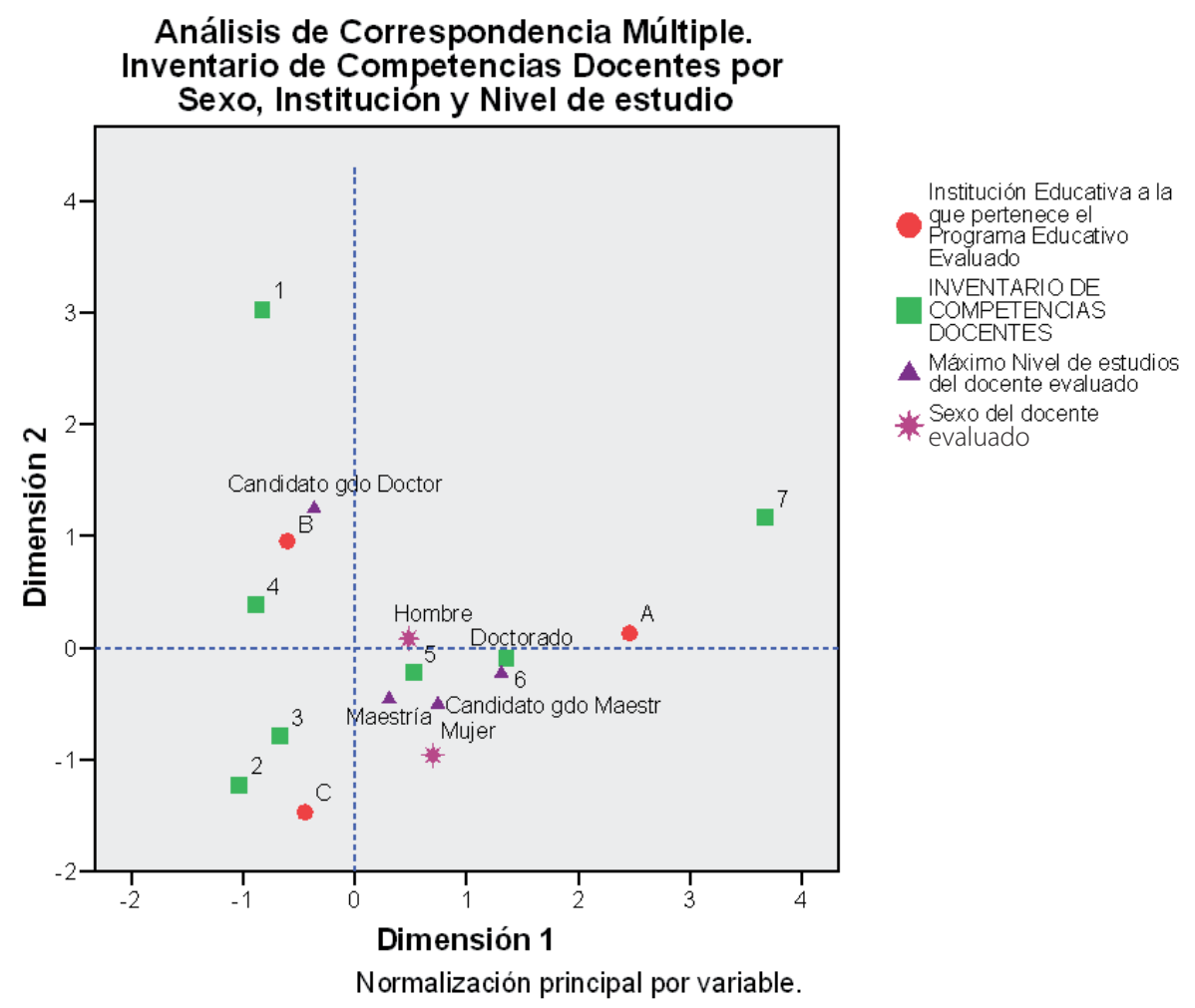

Los análisis realizados a partir de las evaluaciones que los estudiantes hacen a los docentes de los posgrados, permiten identificar competencias aún y cuando no hayan participado en procesos formativos con este enfoque. Estos resultados permiten ubicar cuáles de ellas requieren fortalecerse y cuáles desarrollarse para conformar el perfil docente de los posgrados de las IFD.

\section{Conclusiones}

En la actualidad, persiste el debate sobre las competencias por las implicaciones que subyacen al propio concepto en su versión restringida, dado que se cuestiona el hecho de fomentar la competitividad entre los seres humanos, contrario a las tesis humanistas que plantean la importancia de la solidaridad, el trabajo en equipo y las alternativas de solución desde quienes viven los procesos sociales. Las tendencias actuales en educación y formación profesional, están sustentados en el enfoque basado en competencias lo cual constituye más que una moda, la oportunidad para revisar los resultados de la formación integral de las personas.

Ubicar el desarrollo de competencias en el ámbito educativo, particularmente en el nivel 
superior requiere de una postura amplia anclada a los elementos que la constituyen: capacidades, movilización, recursos cognitivos, contextos específicos. La función de la docencia no se circunscribe a la enseñanza en el sentido de transmisión de conocimientos; como práctica social requiere de una adecuada comunicación, de docentes empáticos tomando en cuenta, que en los posgrados de las IFD, los estudiantes también son profesores, es decir puede hablarse de una relación pedagógica entre iguales, pues viven situaciones semejantes a los docentes de las instituciones de nivel superior. Esta es una distinción peculiar importante de la interacción pedagógica.

El acercamiento teórico a las competencias hace posible identificar la preocupación de académicos e investigadores de vincular la teoría con la práctica. Siendo una tendencia actual, en este trabajo se hace un esfuerzo por configurar una propuesta que retome estos elementos así como las experiencias y conocimientos que surgen de los procesos de evaluación de la docencia que han permitido identificar aciertos y dificultades para constituirse en estrategias de mejoramiento educativo.

Un elemento central que se identificó en la construcción de procesos de evaluación fue la participación de docentes y estudiantes, la técnica del grupo de expertos brindó la posibilidad de incorporar la visión de los participantes y de esta manera conjuntarla con los referentes teóricos y los modelos de la Ried.

Es importante resaltar que las competencias construidas, hacen alusión al contexto y cultura institucional, a los procesos de planeación, desarrollo de la enseñanza, evaluación de aprendizajes, formación continua, interacción pedagógica, comunicación educativa, es decir comprenden situaciones que dimensionan la complejidad de la práctica docente y que hacen necesario incorporar la visión de otros actores para integrar una evaluación integral del desempeño docente.

Las etapas realizadas en este estudio constituyeron una serie de acciones, análisis y reflexión en sí mismas, pero al mismo tiempo dieron pauta para la realización e interrelación de las etapas subsecuentes. En lo concerniente a la caracterización de la docencia en las IFD, los rasgos del perfil docente permitieron dar rostro a los docentes en tanto seres sociales que en la trayectoria conforman su identidad profesional la cual se refleja en la forma de desarrollar la docencia.

Esta experiencia docente se reflejó en el grupo de expertos: sus aportaciones para la identificación de las competencias docentes, denotan sus conocimientos, saberes y visiones acerca del significado de la docencia en las instituciones de educación superior y particularmente en los posgrados en los que han desempeñado diversas funciones administrativas, de gestión, académicas y docentes. Referente a la evaluación por institución educativa, existen diferencias significativas en los resultados obtenidos en una institución respecto de las dos restantes, en la mayoría de las competencias. Esto denota que aun y cuando se tienen programas de estudio similares, perfiles docentes semejantes, la evaluación de las situaciones de docencia que comprenden las competencias, es diversa dado que una característica de la práctica docente es su dimensión personal que la hace única, aún con las semejanzas pedagógicas y didácticas de su realización. 
Siendo esta una primera etapa de validación, las conclusiones pueden considerarse preliminares, en ese sentido, las posibilidades reales del instrumento responden inicialmente a la descripción integral de los aspectos generales evaluados y de las comparaciones establecidas. Por ello:

- El instrumento tiene validez desde el punto de vista conceptual y de contenido, porque las situaciones de docencia planteadas, evalúan eventos significativos en la práctica docente de los profesores de posgrado que son externados mediante desempeños docentes.

- El instrumento puede ser utilizado para los fines de evaluación de estas tres competencias docentes.

- La opciones de aplicación y los criterios para su operación son sencillos y de fácil comprensión. Es un instrumento autoadministrable

- El instrumento puede emplearse en investigaciones posteriores, con el propósito de afinarlo y adecuarlo a necesidades de otros contextos de este nivel educativo. Conforme se avance en su aplicación, relacionando este primer acercamiento con otros contextos educativos, podrán adecuarse las características de su aplicación y las escalas de medición que permitan realizar juicios valorativos desde otras perspectivas.

- Los resultados del piloteo constituyen un punto de partida para identificar áreas de oportunidad desde las cuales, a través del análisis y reflexión de los docentes que desempeñan su función en los posgrados, es posible generar estrategias de formación encaminadas a mejorar el desempeño docente tomando en cuenta las condiciones institucionales y las políticas de educación superior que hoy en día se hacen presentes en la vida académica de las IFD.

Una limitación de este trabajo es que no se trabajaron todas las competencias que por su esencia se llevan a cabo en otros espacios fuera del aula y esta característica deriva en formas de evaluación distintas: autoevaluación, evaluación de coordinadores y pares como refieren Loredo y Rigo (2001). Arribar a la construcción de las competencias docentes en los posgrados de las IFD, de una manera colegiada, considerando las diferencias de las dinámicas institucionales, permitió el enriquecimiento de la discusión y reflexión sobre la práctica docente, lo cual constituye una gran riqueza porque se conjuntaron semejanzas y visiones que permiten el fortalecimiento y colaboración interinstitucional.

Indudablemente que la definición de competencias docentes y su evaluación, requieren de su ubicación contextual y de la participación de los colectivos docentes para lograr el reconocimiento de saberes y al mismo tiempo la identificación de conocimientos, habilidades y actitudes que pueden mejorar vinculando la experiencia docente con procesos de desarrollo y formación profesional.

\section{Bibliografía}

\footnotetext{
García, C. B., Loredo, E. J., Luna, S. E. y Rueda B. M. (2008) Modelo de evaluación de competencias docentes para la educación media y superior. En: IV Coloquio Iberoamericano sobre evaluación de la docencia. Universidad Nacional Autónoma de México, Instituto de Investigaciones sobre la Universidad y RIED. México.
} 
Gorodokin, I. C. (2008) La formación docente y su relación con la epistemología. Revista Iberoamericana de Educación (ISSSN. 1681-56531). México.

Hernández, F. et al. (2005) Aprendizaje, competencias y rendimiento en educación superior. Editorial la Muralla, España.

Ibañez, C. (2007) Metodología para la planeación de la educación superior. Una aproximación desde la Psicología Interconductual. Colección Manuales de Prácticas. Universidad de Sonora. División de Ciencias Sociales. Departamento de Psicología y Ciencias de la Comunicación. México.

Loredo E. J. y Rigo L. (2001) La evaluación docente en un contexto universitario: una propuesta formativa y humanista, en Evaluar para comprender y mejorar la docencia en la educación superior. Cultura universitaria/ serie ensayo, primera edición. México.

Marín, U. R. (2003) El modelo educativo de la UACH. Elementos para su construcción. UACH, México.

Medina M. P. (2000) ¿Eres maestro normalista y/o profesor universitario? La docencia cuestionada, México, Plaza y Valdés.
Mertens, L. (1997) Competencia laboral: sistemas, surgimiento y modelos. México, CONOCER-CINTRFOR/OIT.

Rueda, B. M. (2008) La evaluación del desempeño docente en las universidades públicas en México. IV Coloquio Iberoamericano sobre la evaluación de la docencia. Memorias. Universidad Nacional Autónoma de México. Instituto de Investigadores Sobre la Universidad y la Educación. Red de Investigadores sobre Evaluación de la Docencia. México.

Vargas, F. (2002) Competencia en la formación y competencia en la gestión del talento humano. Convergencias y desafíos. CINTERFOR/OIT, www.cinterfor.org.uy

Zabalza, M. (2007) Competencias docentes del profesorado. Calidady desarrollo profesional. Madrid: Narcea.

Zabala, A. y Arnau, L. (2008) Enseñar competencias comporta partir de situaciones y problemas reales. En: 11 Ideas clave: como aprender y enseñar competencias. Ed. Graó, $4^{a}$ reimpresión 2008. Barcelona España. ISBN: 978-84-7827-500-7 
